\title{
Ampharetidae (Annelida: Polychaeta) from Japanese waters. Part IV. Miscellaneous genera - CORRIGENDUM
}

\author{
MICHAEL REUSCHER, DIETER FIEGE AND MINORU IMAJIMA
}

doi: http://dx.doi.org/10.1017/So025315415000545. Published by Cambridge University Press: 20 May 2015

Keywords: Ampharetidae, Amage, Amphisamytha, Glyphanostomum, Lysippe, Melinnopsis, Orochi, Samytha, new genus, new species, corrigendum

The species names of the four species Amage auricula, Ampharete falcata, Auchenoplax crinita and Lysippe labiata were incorrectly identified as Amage abiater, Ampharete abiate, Auchenoplax abiate and Lysippe abiate, respectively in Table 1. Please see below for the correct version of the table.

\section{REFERENCE}

Michael Reuscher, Dieter Fiege and Minoru Imajima. Ampharetidae (Annelida: Polychaeta) from Japanese waters. Part IV. Miscellaneous genera. Journal of the Marine Biological Association of the United Kingdom, available on CJO2015. doi:10.1017/Soo25315415000545. 
Table 1. List of all Ampharetidae species recorded from Japan, along with references of the records. Abbreviation: OD, original description.

\begin{tabular}{|c|c|}
\hline Species & Reference \\
\hline \multicolumn{2}{|l|}{ Ampharetinae } \\
\hline Amage cf. adspersa (Grube, 1863) & This study \\
\hline Amage auricula Malmgren, 1866 & $\begin{array}{l}\text { von Marenzeller (1884), Hessle (1917), Imajima and Hartman (1964), } \\
\text { Imajima }(1997,2009)\end{array}$ \\
\hline Amage delus (Chamberlin, 1919) & Imajima (2009) \\
\hline Amage ehlersi sp. nov. & This study \\
\hline Amage longitorus sp. nov. & This study \\
\hline Amage scutata Moore, 1923 & Imajima (2009) \\
\hline Ampharete ampullata Imajima et al., 2012 & OD \\
\hline Ampharete cinnamomea Imajima et al., 2012 & OD \\
\hline Ampharete falcata Eliason, 1955 & Imajima et al. (2012) \\
\hline $\begin{array}{l}\text { Ampharete finmarchica (Sars, 1865) [including junior synonym } A \text {. } \\
\text { arctica Malmgren, 1866] }\end{array}$ & $\begin{array}{l}\text { Okuda (1936), Imajima and Hartman (1964), Imajima (1968, 1988, } \\
\text { 1992, 1994, 2011) }\end{array}$ \\
\hline Ampharete kudenovi Jirkov, 1994 & $\mathrm{OD}$ \\
\hline Ampharete lindstroemi Hessle, 1917 & Imajima et al. (2012) \\
\hline Ampharete longipaleolata Ushakov, 1950 & Imajima (2009) \\
\hline Ampharete orientalis (Annenkova, 1929) & Imajima et al. (2012) \\
\hline $\begin{array}{l}\text { Amphicteis gunneri (Sars, 1835) [including junior synonym } A \text {. } \\
\text { japonica McIntosh, 1885] }\end{array}$ & $\begin{array}{l}\text { McIntosh (1885), Moore (1903), Hessle (1917), Okuda (1938), } \\
\text { Takahashi (1938), Imajima and Hartman (1964), Imajima (1988, } \\
\text { 1997, 2001) }\end{array}$ \\
\hline Amphicteis mederi Annenkova, 1929 & Imajima $(2009,2011)$ \\
\hline Amphicteis scaphobranchiata Moore, 1906 & Imajima (2009) \\
\hline Amphicteis spinosa Reuscher et al., 2014 & $\mathrm{OD}$ \\
\hline Amphicteis taurus Reuscher et al., 2014 & $\mathrm{OD}$ \\
\hline Amphicteis uncopalea Chamberlin, 1919 & Reuscher et al. (2014) \\
\hline Amphisamytha japonica Hessle, 1917 & OD, Imajima and Hartman (1964), Imajima (2009), This study \\
\hline Anobothrus dayi Imajima et al., 2013 & OD \\
\hline Anobothrus fimbriatus Imajima et al., 2013 & $\mathrm{OD}$ \\
\hline Anobothrus flabelligerulus Imajima et al., 2013 & OD \\
\hline Anobothrus gracilis (Malmgren, 1866) & Imajima $(2009,2011)$ \\
\hline Anobothrus mironovi Jirkov, 2009 & $\mathrm{OD}$ \\
\hline Anobothrus patersoni Jirkov, 2009 & OD \\
\hline Anobothrus wakatakamaruae Imajima, 2009 & $\mathrm{OD}$ \\
\hline Auchenoplax crinita Ehlers, 1887 & Imajima $(1997,2009,2011)$ \\
\hline Glyphanostomum hesslei sp. nov. & This study \\
\hline Glyphanostomum pallescens (Theel, 1879) & This study \\
\hline Lysippe fragilis (Wollebaek, 1912) & Hessle (1917), This study \\
\hline Lysippe labiata Malmgren, 1866 & Imajima $(2009,2011)$ \\
\hline Lysippe nipponica sp. nov. & This study \\
\hline Orochi palacephalus gen. et sp. nov. & This study \\
\hline Paramphicteis angustifolia (Grube, $1878 \mathrm{c}$ ) & Reuscher et al. (2014) \\
\hline Paramphicteis weberi (Caullery, 1944) & $\begin{array}{l}\text { von Marenzeller (1884), Imajima and Hartman (1964), [both as } \\
\text { Amphicteis angustifolia], Reuscher et al. (2014) }\end{array}$ \\
\hline Phyllocomus hiltoni (Chamberlin, 1919) & Imajima (2011) \\
\hline Samytha annenkovae sp. nov. & This study \\
\hline Samytha oculata Grube, 1878 a & OD \\
\hline Samythella bathycola Uschakov, 1950 & Imajima (2009) \\
\hline Samythella neglecta Wollebaek, 1912 & Imajima (2009) \\
\hline Samythella pacifica (McIntosh, 1885) & OD \\
\hline Sosane brevibranchiata Imajima et al., 2013 & OD \\
\hline Sosane cf. cinctus (Hartman, 1965) & Imajima et al. (2013) \\
\hline Sosane sulcata Malmgren, 1866 & Hayashi and Hanaoka (1997), Imajima (2006), Imajima et al. (2013) \\
\hline Sosane trigintaduo Imajima et al., 2013 & OD \\
\hline Sosane uebelackerae Imajima et al., 2013 & OD \\
\hline Sosane wireni (Hessle, 1917) & Imajima et al. (2013) \\
\hline Tanseimaruana boninensis Imajima et al., 2013 & $\mathrm{OD}$ \\
\hline Watatsumi grubei Reuscher et al., 2014 & OD \\
\hline Zatsepinia jirkovi Imajima et al., 2013 & OD \\
\hline Zatsepinia rittichae Jirkov, 1986 & Imajima et al. (2013) \\
\hline \multicolumn{2}{|l|}{ Melinninae } \\
\hline Melinna cristata (Sars, 1851) & Imajima $(1997,2009,2011)$ \\
\hline Melinna elisabethae McIntosh, 1914 & Imajima $(1994,2009,2011)$ \\
\hline Melinnopsis augeneri sp. nov & This study \\
\hline Melinnopsis mcintoshi sp. nov. & Imajima (2009) [as M. atlantica McIntosh, 1885], this study \\
\hline Melinnopsis tetradentata (Imajima, 2001) & OD, Imajima (2011) \\
\hline
\end{tabular}

